\title{
Correlation of Microstructure and Properties of Cold Gas Sprayed INCONEL 718 Coatings
}

\author{
R. Vaßen ${ }^{1}$ - J. Fiebig ${ }^{1} \cdot$ T. Kalfhaus ${ }^{1} \cdot$ J. Gibmeier $^{2} \cdot$ A. Kostka $^{3} \cdot$ \\ S. Schrüfer ${ }^{4}$
}

Submitted: 30 October 2019/in revised form: 21 January 2020/Published online: 3 February 2020

(C) The Author(s) 2020

\begin{abstract}
In the cold gas spray process, deposition of particles takes place through intensive plastic deformation upon impact in a solid state at temperatures well below their melting point. The high particle impact velocities and corresponding peening effects can lead to high compressive residual stresses in cold spray coatings. This can be advantageous with regard to mechanical properties as fatigue life and hence, cold spray is an ideal process for repair applications. In this study, INCONEL 718 particles were cold sprayed by using nitrogen as propellant gas. The deposited coatings with different thicknesses were characterized using electron microscopy techniques to study grain refinement and precipitates in the coating. In addition, depth-resolved residual stress measurements have been performed by the incremental hole drilling method.
\end{abstract}

This article is part of a special topical focus in the Journal of Thermal Spray Technology on Advanced Residual Stress Analysis in Thermal Spray and Cold Spray Processes. This issue was organized by Dr. Vladimir Luzin, Australian Centre for Neutron Scattering; Dr. Seiji Kuroda, National Institute of Materials Science; Dr. Shuo Yin, Trinity College Dublin; and Dr. Andrew Ang, Swinburne University of Technology.

R. Vaßen

r.vassen@fz-juelich.de

1 IEK-1, Forschungszentrum Jülich GmbH, 52425 Jülich, Germany

2 Institut für Angewandte Materialien - Werkstoffkunde (IAMWK), Karlsruher Institut für Technologie, KIT-Campus Süd, 76131 Karlsruhe, Germany

3 Zentrum für Grenzflächendominierte Höchstleistungswerkstoffe (ZGH), Ruhr-Universität Bochum, 44801 Bochum, Germany

4 Rolls-Royce Deutschland Ltd \& Co KG., 15827 Blankenfelde-Mahlow, Germany
The residual stress depth profiles in the coatings indicate compressive residual stresses of several hundred MPa which are hardly influenced by the coating thickness. In addition, large compressive stress levels are found in surface-near regions of the substrate due to the grit blasting process. Furthermore, a post-heat treatment analysis was performed to investigate its influence on residual stresses and bonding strength. These findings are used to develop a consistent explanation of the dependence of strength values on thickness.

Keywords Cold gas spraying - Electron microscopy INCONEL 718 . Incremental hole drilling method . Residual stress

\section{Introduction}

Cold gas spraying has become an important thermal spray process with numerous applications (Ref 1-3). A huge number of different materials reaching from pure metals to alloys and composites have been successfully deposited by this process reaching low porosity levels (Ref 4). The basic deposition mechanisms have been identified as a confined deformation, the so-called adiabatic shear instability, which leads to a removal of oxide scales, good intermixing and finally mechanical bonding of the deposited particles even without melting (Ref 5). This bonding mechanism implies that only in a certain process window, the deposition is efficient. Typically, the velocity of the particles at the impingement on the substrate has to be above a socalled critical velocity $\mathrm{v}_{\mathrm{cr}}$, otherwise the adiabatic shear instability will not be efficient (Ref 6). If the velocities become too high, erosion of the substrate will take place (Ref 5). The critical velocity is typically reduced with an 
increase in particle temperature. The deposition of highstrength alloys often needs the preheating of the particles to reach the critical velocity. Meanwhile, systems with preheating of the propellant gas above $1000{ }^{\circ} \mathrm{C}$ are available.

As the cold spray deposition allows the manufacture of dense, metallic layers at moderate substrate temperatures and also rather low particle temperatures, a low oxygen uptake and hence high purity of the deposits results even for sensitive materials (Ref 7). In addition, the fast impact leads to the generation of compressive stress levels in the coatings (Ref 8), which are typically beneficial for the mechanical properties as fatigue. Hence, the process is rather ideal for repair applications and many efforts have been made in this direction (Ref 9, 10). For the repair application, INCONEL 718 is an interesting material as it is often used in aircraft components (Ref 11). Several investigations have been made on the deposition of INCONEL 718 by cold spray processes (Ref 12-14) demonstrating that INCONEL 718 parts can be successfully manufactured by cold spray. In a recent publication, additions of a martensitic stainless steel have been used to obtain high-density coatings (Ref 15). Further recent work shows that the manufacture of highly dense INCONEL 718 coatings by the cold spray process is possible especially by using high pressures up to $7 \mathrm{MPa}$ and high propellant gas temperatures of $1000{ }^{\circ} \mathrm{C}$ (Ref 16). Also high tensile strengths of up to $133 \mathrm{MPa}$ could be obtained. In contrast to our paper, no discussion on coating thickness, stress levels, and microstructural features especially phase evaluation is given.

A very recent publication (Ref 17) on cold gas sprayed INCONEL 718 focuses on the optimization of the spray process. It also covers residual stress profiles in these coatings, and the results will be compared to our results.

The determined compressive residual stresses in the coatings might lead to an impact on the coating's performance. In Ref 18, a reduction in adhesion strength of cold sprayed coatings with increasing coating thickness was found and explained with the increasing stored energy in thicker coatings which promote failure. However, in this paper, it was not shown if the microstructure of the coatings changed with coating thickness and had an additional influence on this finding. In order to better understand the origin of the drop of strength with coating thickness, coatings with three different thickness values have been prepared in the present investigation and characterized in depth with respect to microstructure and residual stress state.

\section{Experimental Procedures}

\section{Sample Preparation}

For the coating manufacture, a powder based on the INCONEL 718 composition (Oerlikon-Metco, Troy, MI, USA) with spherical morphology, and a mean particle size of $14 \mu \mathrm{m}$ was used as feedstock powder [more details are found in Ref 19]. Figure 1 reveals the details of the microstructure of the powder used in this study. The bright contrast corresponds to interdendritic regions where $\mathrm{Nb}$ is preferentially segregating due to the fast cooling rate during the gas atomization process used for the powder manufacture. It should be mentioned here that the powder contained a certain amount of additional nickel-aluminumrich phase. This addition corresponds to a patent application filed by Oerlikon in which the blending of a softer, shear-deformable, secondary-phase metal and/or metal alloy in a nickel- or iron-based material for the deposition of a dense coating is claimed ( $\operatorname{Ref} 20$ ). As will be seen later, this phase is visible in the microstructure of the coatings.

The substrates for all tests were disk shaped with $25 \mathrm{~mm}$ in diameter and a thickness of 5 or $6.5 \mathrm{~mm}$. The substrates were grit-blasted with commercially available alumina grit with an average particle size of $420-600 \mu \mathrm{m}$ and a pressure of 2.5 bar prior to deposition.

In additional experiments, this pressure was identified as an upper pressure limit to guarantee a negligible amount of $\mathrm{Al}_{2} \mathrm{O}_{3}$ particles at the interface. However, still some loading with alumina was observed, an internal investigation showed that other surface preparation techniques as laser ablation can avoid that with even improved bonding. In this investigation, only the more established technique grit blasting was used. The average substrate roughness of

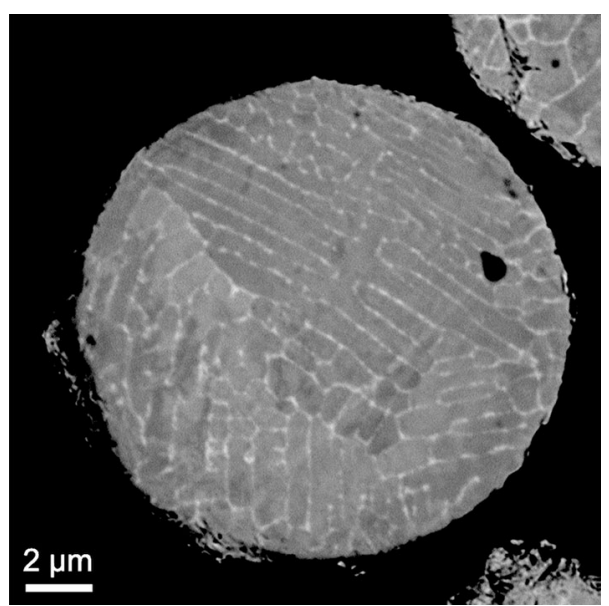

Fig. 1 SEM (BSE) image showing a cross section of the used IN718 powder 
Fig. 2 SEM micrographs of the thin (a), medium (b), and thick (c) coatings

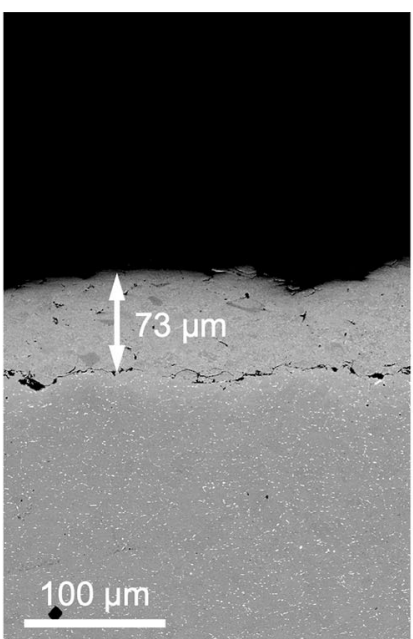

(a)

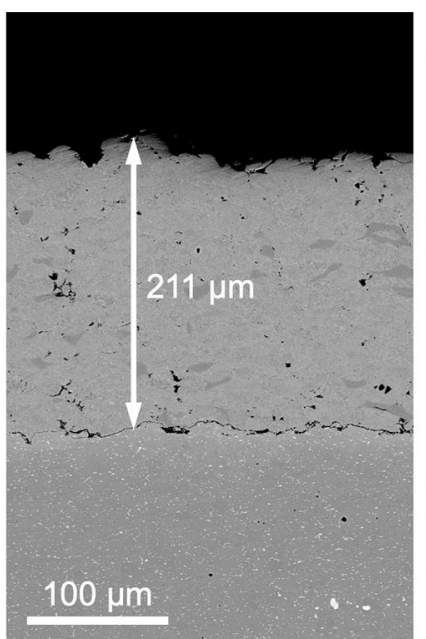

(b)

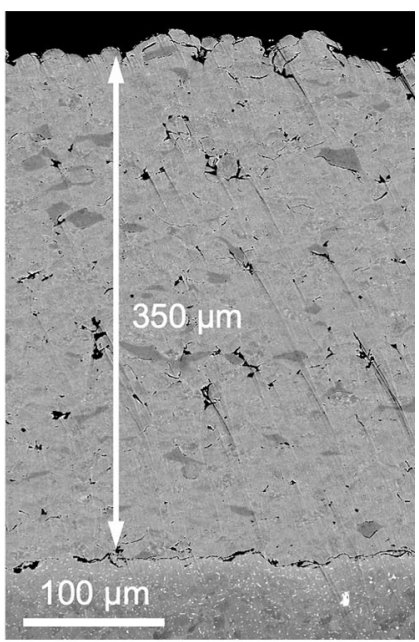

(c)

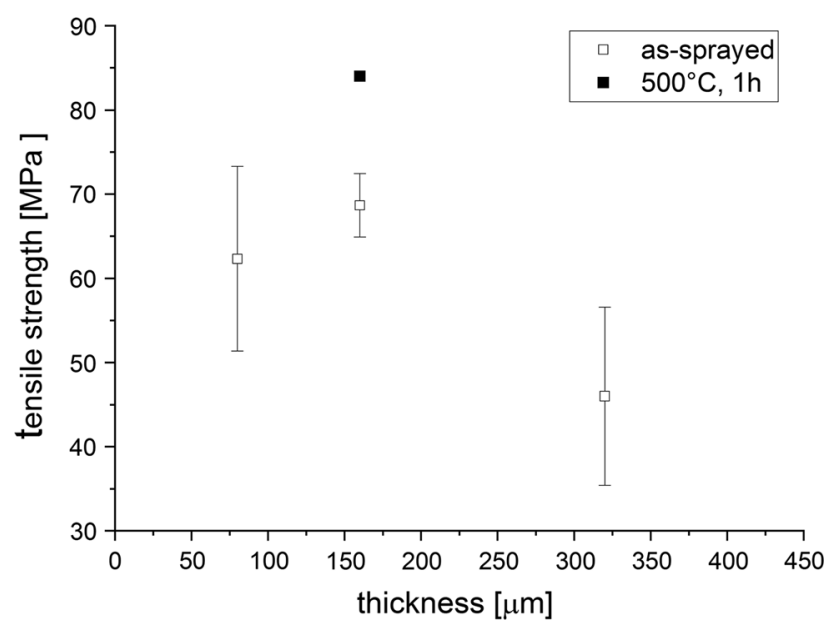

Fig. 3 Tensile strength of the cold sprayed coatings as a function of thickness. In addition, the strength after annealing at $500{ }^{\circ} \mathrm{C}, 1 \mathrm{~h}$, is given for the medium thickness coating

$R_{\mathrm{a}}(\approx 3.1 \mu \mathrm{m})$ was evaluated by using an optical profilometer (Model CT350T, cyberTECHNOLOGIES $\mathrm{GmbH}$, Germany).

For the deposition of the cold sprayed INCONEL 718 coatings, a CGT-Oerlikon-Metco Kinetics ${ }^{\circledR} 8000$ highpressure cold spray system equipped with the standard water-cooled D-24 de-Laval-type converging-diverging nozzle was used with only nitrogen as propellant gas. The inlet gas pressure and the temperature were $4 \mathrm{MPa}$ and $950{ }^{\circ} \mathrm{C}$, respectively. The stand-off distance from the nozzle exit to the substrate surface was $60 \mathrm{~mm}$. A spray angle of $90^{\circ}$ was used for the coating deposition. More details are given in Ref 21. Coating thickness was varied by varying the number of deposition passes using 1,2 , and 4 passes.

\section{Characterization Methods}

Coating adhesion test was performed on a tensile rig as in the standard test method DIN EN ISO 6892-1 (Ref 22). A tensile load was applied to bonded cylindrical slugs, using a constant rate of $1 \mathrm{~mm} / \mathrm{s}$, until fracture occurred, and the amount of force required for separation was recorded. Adhesion strength was determined as the maximum load divided by the cross-sectional area of the specimen. The coated specimen was examined after failure, and failure mode was identified, if the coating failed internally (cohesive failure), the coating separated from the substrate (adhesive failure), or the coating remained intact with the substrate and the epoxy between specimen and slug failed. For each thickness, three samples were tested.

The residual stress depth profiles within the coatings and the substrates were evaluated using the incremental hole drilling method (Ref 23-25). For the application of the incremental hole drilling technique, a blind hole is incrementally drilled into the material while the strain release on the surface is measured using strain gauges. From the measured strain relaxations, the corresponding residual stresses are calculated on the basis of calibration data and the material elastic constants. The incremental hole drilling was carried out using a self-constructed drilling device and a TiN cement carbide tool with a nominal diameter of $1.6 \mathrm{~mm}$. The strain relaxations were determined using strain gauge rosettes of type CEA-06-062UM-120, while each individual strain gauge was connected to a carrier frequency amplifier, type Picas, by Peekel Instruments $\mathrm{GmbH}$ (Bochum, Germany) via a half bridge (Wheatstone bridge) circuit with temperature compensation. In addition, in one case, the residual stress depth profile was determined by x-ray diffraction according to the $\sin ^{2} \psi$-method (Ref 26). The residual stress depth distribution was determined 


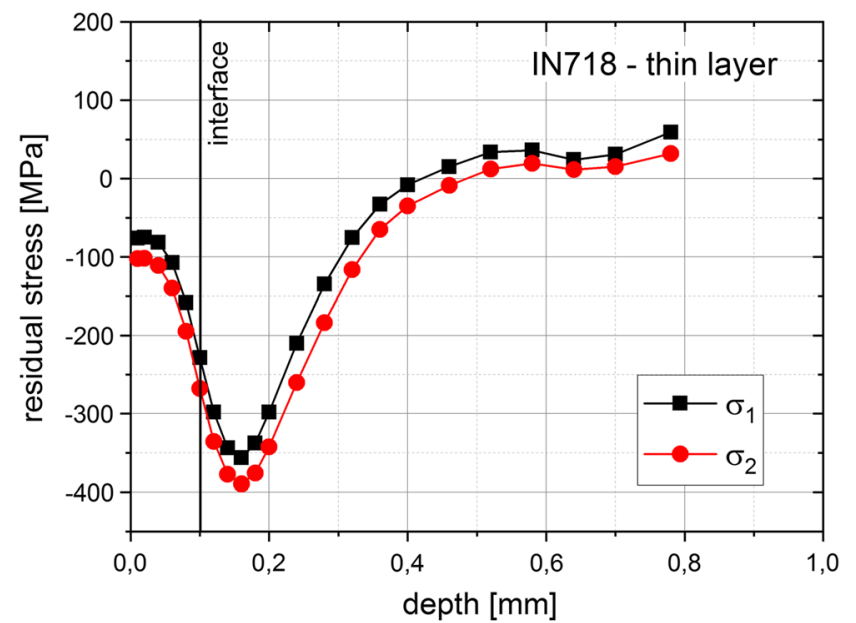

(a)

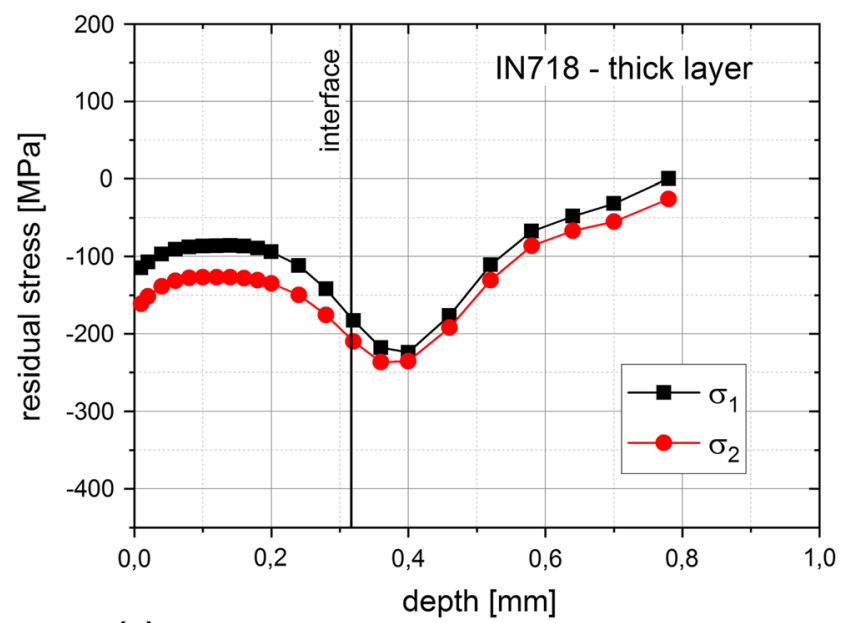

(c)

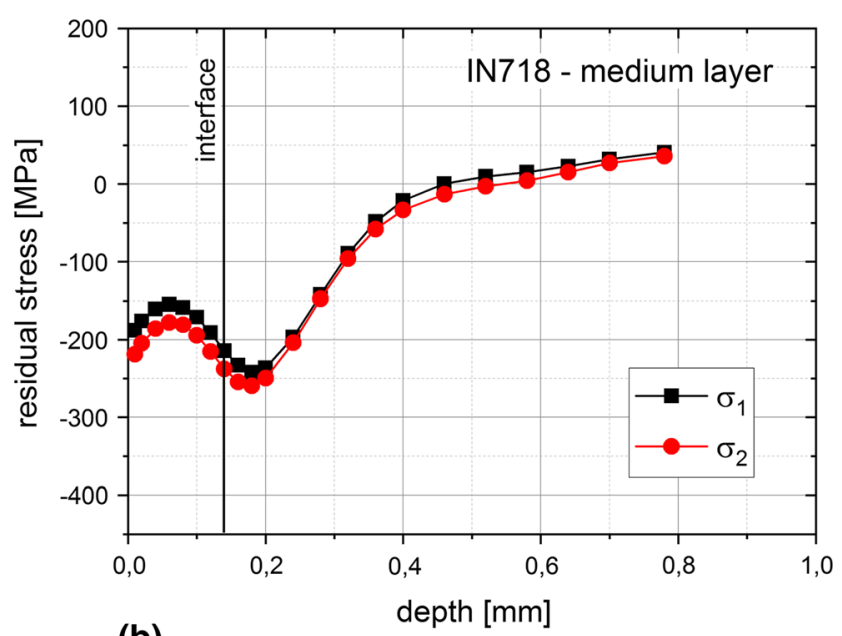

(b)

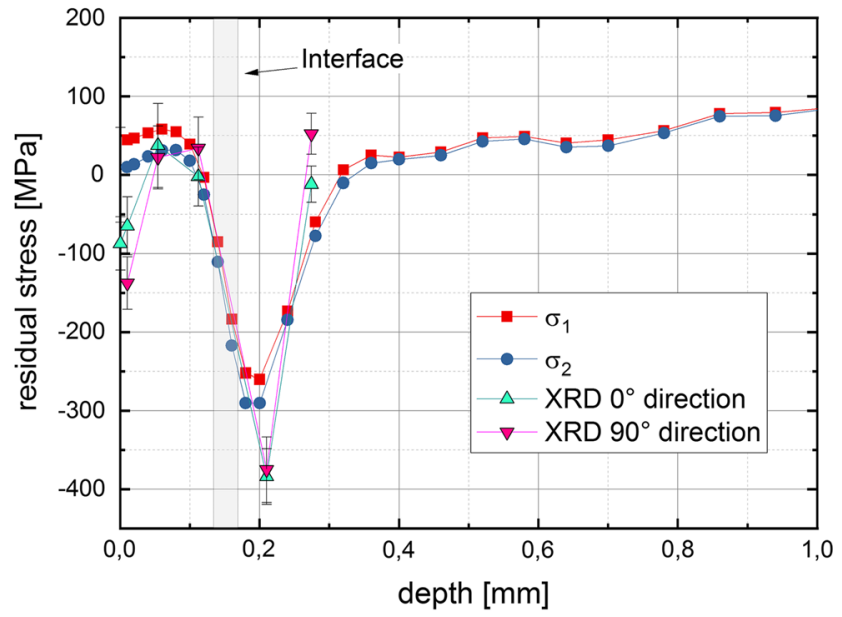

(d)

Fig. 4 Stress profile of the three as-sprayed (thin a, medium b, thick c) and the annealed $\left(\mathrm{d}, 500{ }^{\circ} \mathrm{C}, 1 \mathrm{~h}\right)$ coating

by reapplication of $\mathrm{x}$-ray stress analysis on the newly generated surface after defining successive electrochemical layer removal. The $\mathrm{x}$-ray residual stress analysis was carried out for the $\{220\}$ diffraction lines of the $\gamma-\mathrm{Ni}$ phase using 15 tilt angles $\psi$ in the range between $-60^{\circ} \leq \psi$ $\leq 60^{\circ}$ using $\mathrm{V}$-filtered $\mathrm{CrK} \alpha$-radiation. The primary beam was masked by means of a 2-mm pinhole collimator. On the secondary side, a 4-mm slit was used in front of the scintillation counter. As x-ray diffraction elastic constants, literature data for bulk INCONEL were used.

Coating morphologies were investigated by scanning electron microscopy (SEM, Ultra 55, Zeiss-Germany). Further, microstructural studies were made using SEM and TEM at Ruhr University of Bochum. Specimens for SEM investigations were prepared using a Hitachi IM400PLUS cross-section polisher operating at $4 \mathrm{kV}$. Jeol JSM-7200F instrument operating at $15 \mathrm{kV}$ was applied for imaging and collecting EDX and EBSD data. TEM imaging was performed using a FEI Tecnai Supertwin F20 equipped with high-angle annular dark field (HAADF) detector and operated at $200 \mathrm{kV}$. TEM samples were prepared using FEI Helios Nanolab G4 CX focused ion beam system (FIB) operated at $30 \mathrm{kV}$.

Annealing was performed in a vacuum furnace at $500{ }^{\circ} \mathrm{C}$ for $1 \mathrm{~h}$.

\section{Results and Discussion}

Figure 2 reveals microstructures of the three specimens with coating thickness of 73,211 , and $350 \mu \mathrm{m}$, respectively. The density of all the coatings is high, and porosity levels are low. As discussed later, the dark phases are due to the NiAl additions to the INCONEL 718 particles. Furthermore, a rather high number of cracks appear at the interface. One reason for these cracks are-although a rather low pressure for grit blasting was used-the comparably high amount of alumina particles at the interface 


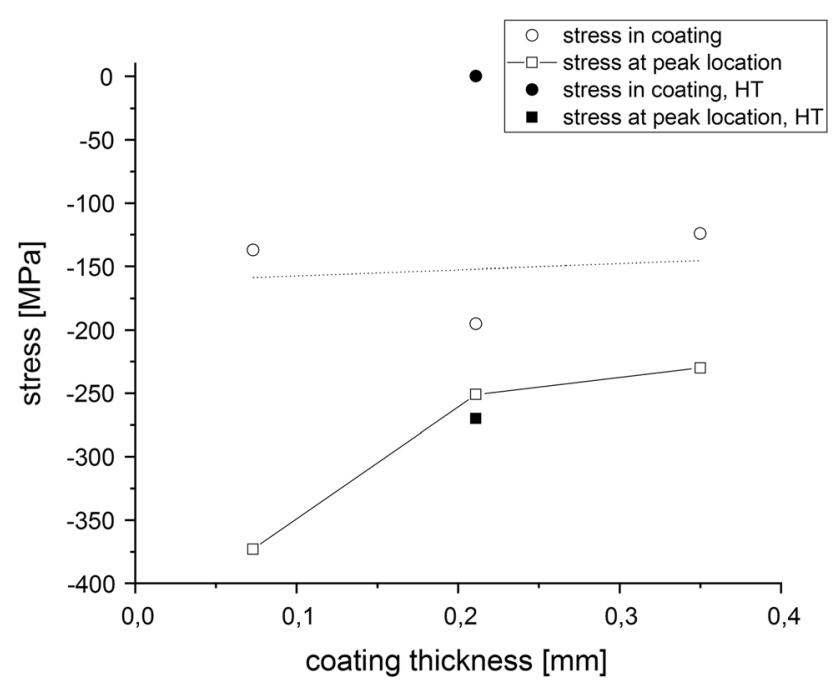

Fig. 5 Mean residual stress in the coatings and at the peak location in the substrate for the coatings with different thicknesses for the assprayed (open symbols) and the annealed $\left(500{ }^{\circ} \mathrm{C}, 1 \mathrm{~h}\right)$ condition (closed symbols). The dotted line indicates a linear fit of the stress in the coating, and the solid lines connect the symbols showing the stress at the peak location

with initiate cracks at this location. These cracks will also lead to a reduction in the adhesion strength.

In Fig. 3, the results of adhesion strength measurements are presented. All coatings in the as-sprayed condition failed at the interface substrate/coating. Although the results show a rather large scattering range, a tendency of reduced strength with increased coating thickness is visible. As stated in the introduction, similar results were found in Ref 18. In addition to the as-sprayed condition, also the strength of the coating with a medium thickness is given after annealing at $500{ }^{\circ} \mathrm{C}$ for $1 \mathrm{~h}$. A clear increase in strength can be observed. Furthermore, the failure mode changed, as the failure now occurred in the glue, indicating that the actual adhesion strength is probably even higher.

For the as-sprayed coatings and the annealed one, the residual stress depth profile has been measured with the incremental hole drilling method, and the results are shown in Fig. 4. Sigma 1 and sigma 2 indicate the two principle stress components for the in-plane stress state.

As an important result, it should be stated that both hole drilling method and XRD analysis gave very similar results as shown in Fig. 4(d). These findings certainly underline the reliability of the hole drilling method. Deviation occur at the very surface, which is due to the fact that (1) the incremental hole drilling method is less sensitive in the very surface-near region and (2) thermally sprayed coatings show a rather large surface topography, which makes it rather difficult to find the zero depth value.

The residual stress depth distribution for the as-sprayed coatings shows compressive residual stress values within the coatings. Compressive residual stress in cold spray coatings is typically found due to the fast impinging of the particles on the substrate (Ref 8 ). In the coatings using 2 (medium-layer thickness, Fig. 4b) and 4 (thick, Fig. 4c) passes, one can observe an increase in the compressive residual stresses at the surface which might be attributed to the consecutive layer and stress buildup as described in Ref 27. This should give a higher stress level at the surface. The highest compressive residual stresses were observed near to the interface to the substrate, i.e., within the surface of the substrates. This is a result of the grit blasting prior to the deposition (Ref 28). The TEM investigation shown later confirms the massive change of the microstructure in this region. Deeper within the substrate, the residual stresses are reduced and are expected to reach tensile values far away from the interface to compensate for the compressive residual stresses in the coatings and the substrate surface. After the heat treatment (Fig. 4d), the residual stress in the coating practically disappeared; however, the residual stress values at the surface of the substrates remained. A summary of the residual stress evaluation is shown in Fig. 5 using mean stress values for the coatings. The mean stress levels in the coatings are quite close, varying between about 120 and $200 \mathrm{MPa}$. So no systematic influence of the thickness is visible. The highest compressive stress level is found for the coating with the medium thickness. The heat treatment reduces the stress to nearly zero. Stress levels at the surface of the substrates induced by the grit blasting were much higher between 230 and $370 \mathrm{MPa}$ and remained rather unchanged during heat treatment.

Also in Ref 17, stress profiles in cold sprayed INCONEL 718 coatings have been made by XRD and layer removal. The used propellant gas pressure was with 50 bar higher, temperatures were 800,900 , and $1000{ }^{\circ} \mathrm{C}$. The compressive stress levels are between 150 and $400 \mathrm{MPa}$, quite close to our results. Rather no systematic influence of the temperature is found. However, in contrast to our observation, an increase in the stress level is observed with an increase in distance from the surface in contradiction to the model prediction in Ref 27. Also no distinct compressive stress peak close to the substrate surface was found. That might be related to the heating of the substrates to $100{ }^{\circ} \mathrm{C}$ during the grit blasting.

In order to get a better insight in these results, further SEM and TEM investigations of the samples have been performed. First, a substrate has been analyzed after grit blasting (Fig. 6a, b, and c). As it is known from the literature, a massive deformation takes place during the grit blasting process, which leads to the high compressive residual stresses at the interface on the substrate surface side. The ultra-fine-grained region formed during grit blasting consists of grains smaller than $50 \mathrm{~nm}$. These grains are elongated in the direction perpendicular to the 
Fig. 6 Comparison of the gritblasted substrate and coated substrate: (a) SEM micrograph of the grit-blasted substrate showing the location of the TEM lamella and $\mathrm{Ni} 3 \mathrm{Nb}$ particles (white contrast) strongly affected by the accumulated strain, (b) BFTEM image of a typical $\mathrm{Ni} 3 \mathrm{Nb}$ particle present in the the gritblasted substrate and corresponding diffraction pattern (inset), (c) HAADFTEM micrograph revealing a part of the deformed region at the surface of the substrate. (d) HAADF-TEM micrograph showing the interface between the grit-blasted specimen and coated specimen (thinnest coating). Dark rectangular contrast particles at the interface refer to the embedded alumina particles
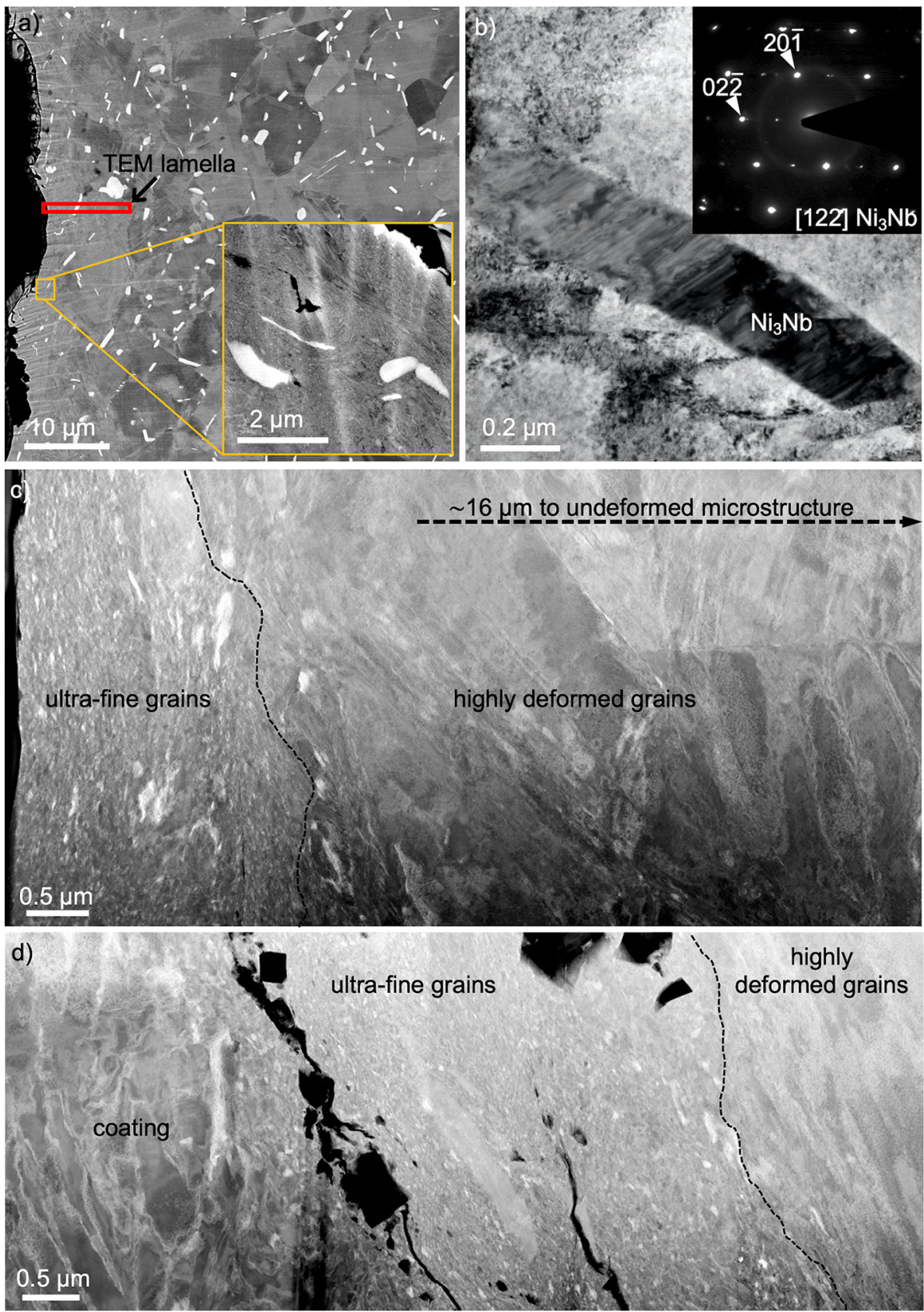

grit-blasted surface. There is also some indication that the $\mathrm{Ni}_{3} \mathrm{Nb}$ particles are partially dissolved (smeared) during the massive deformation (see inset in Fig. 6a). The zone thickness massively influenced by grit blasting is nearly $20 \mu \mathrm{m}$ in thickness.

Figure 6(d) shows, on the left side, the TEM image of the thinnest coating. There are dark areas in the size range of about half a micrometer surrounded irregularly by bright phases. This microstructure can be explained by the microstructure of the powder (see Fig. 1) in which Nb-rich (bright) areas are surrounding the dendritic regions with less $\mathrm{Nb}$. Due to the cold spray process, these regions have been deformed and the well-aligned structure in the powder is destroyed. The part of the coating shown in Fig. 6(d) is probably part of one former particle. As shown later in EBD results, the particles are only deformed largely in a shell region. This is indicated by the fine-grained, about half a micron thick region of the coating close to the interface, which is indicated by the alumina particles.

In Fig. 7(a), a SEM analysis of the thickest coating is shown. The results are similar for the other coatings; therefore, only one type of coating is shown here. Figure 7(a) represents a low magnification overview in which the complete coating, substrate, and the interface are 

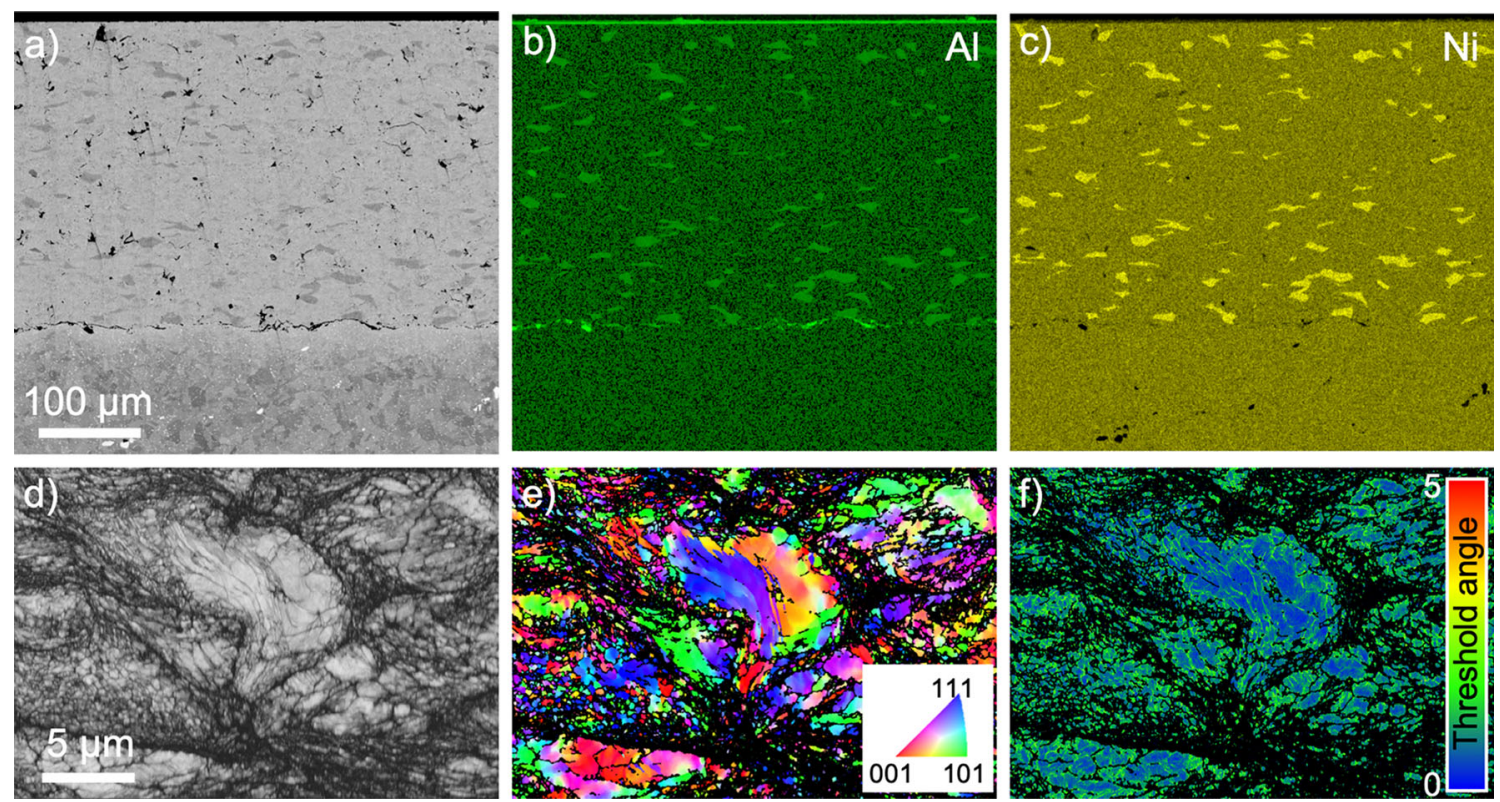

Fig. 7 SEM analysis of the sample with the thickest coating. (a) BSE micrograph, (b) Al map, (c) Ni map, (d) EBSD image quality map showing the presence of very fine grains in the outer region of the

particles; (e) IPF Z map showing grain orientation and (f) Kernel average misorientation (KAM) map revealing the degree of accumulated strain (Color figure online)

Fig. 8 BSE micrograph showing Al-Ni inclusion in the IN718 coating and corresponding EDX maps. Red rectangle shows the region from which a TEM lamella shown in Fig. 10 was taken (Color figure online)

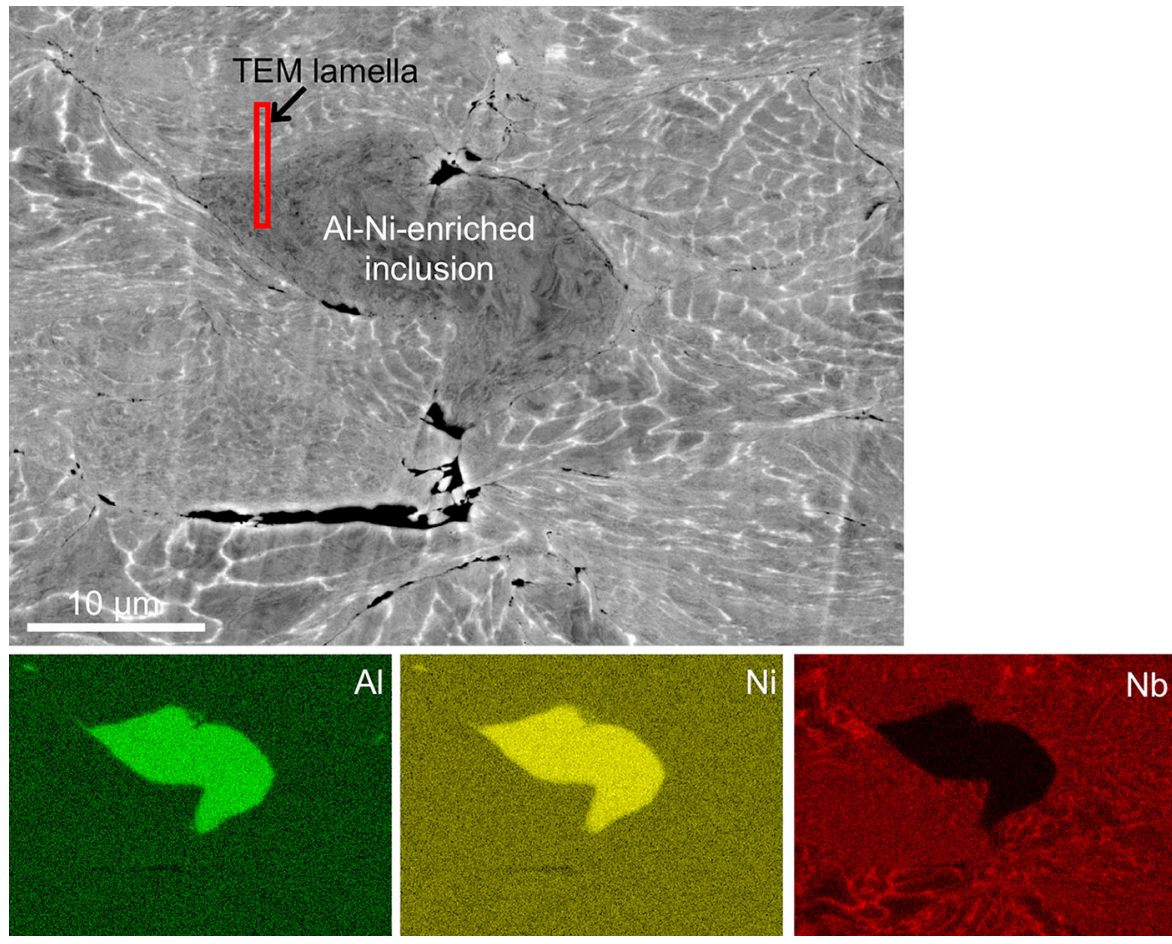

shown. Corresponding element distribution maps (Fig. 7b and c) clearly reveal the presence of regions enriched with $\mathrm{Al}$ and $\mathrm{Ni}$ within the coating. These features are due to additions of NiAl-rich phases in the spray powders to improve the deformation behavior and hence the deposition efficiency of the powder. Figure 7(d), (e), and (f) shows a high-resolution EBSD data acquired from the coating. The maps reveal that the whole region is heavily deformed; however, the center of the particles consists of larger and less deformed grains. The color-coded IPF Z map (Fig. 7e) shows the presence of low-angle grain boundary within the particle, and the Kernel average misorientation map (Fig. 7f) reveals that most of the strain is accumulated at the fine-grained regions outside. This microstructure is due 
to the cold spray process in which only the periphery of particles is heavily deformed, while the core remains rather unchanged. During the spray deposition, dynamic recrystallization can occur which leads to the observed fine grain structure. This was also previously shown, e.g., in cold sprayed Ni coatings (Ref 29).

Figure 8 reveals interesting features in the cold sprayed INCONEL 718 regarding the nickel-aluminum-rich phase added to the powder visible (see above). An inclusion consisting of this phase is visible in the center of the figure. Although it is expected that these additions give an improved deformation behavior during cold spray, also some cracks appear at the interface to the INCONEL 718 matrix, which might reduce mechanical properties. The second interesting feature is the fine-grained microstructure of the matrix. It is separated by bright contrast areas which are probably the remainings of the interdendritic areas present in the particles (see Fig. 1). EDX maps reveal that these areas are highly enriched by $\mathrm{Nb}$. These findings correspond to the TEM investigation shown in Fig. 6. Significantly different local compositions might result in local stresses that explain the presence of some cracks.
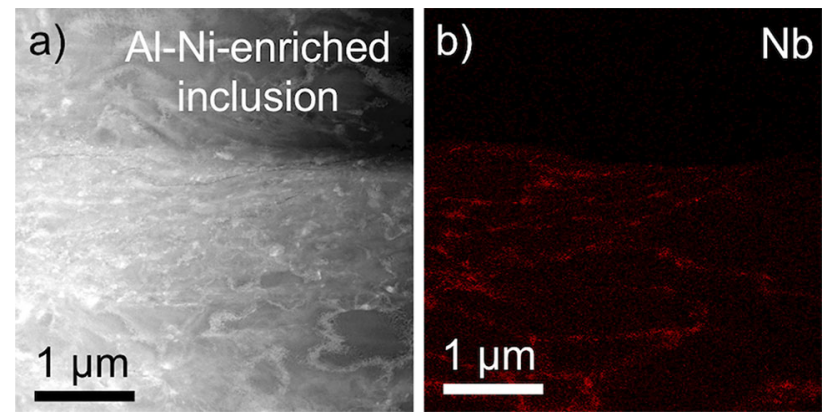

Fig. 9 TEM investigation of the interface between the Al-Nienriched inclusion and the material of the coating (a); Corresponding EDX map showing bright segregation of $\mathrm{Nb}$ into former interdendritic regions of the coating. $\mathrm{No} \mathrm{Nb}$ is detected within the $\mathrm{Al}-\mathrm{Ni}$ inclusion
Figure 8 also shows that the cracks do not follow the Nbenriched GB's.

A more detailed investigation of the interface between the INCONEL 718 matrix and the Al-Ni-enriched inclusion was made by TEM-HAADF investigation. Figure 9(a) shows such a micrograph. The micrograph only partly confirms the already discussed results; at the interface, the corresponding Nb EDX map (Fig. 9b) shows that the $\mathrm{Nb}$ is strongly segregated into defined areas in the coating. However, in contrast to the SEM results, in the TEM investigation, no cracks are found at the interface; instead, a good bonding is observed.

Figure 10 compares the microstructure of the interface region coating/substrate in the as-sprayed state and after annealing $\left(1 \mathrm{~h}\right.$ at $\left.500{ }^{\circ} \mathrm{C}\right)$. It is clearly visible that the grains of the coating significantly coarsened, while the substrate does not change too much. This indicates that recovery processes are already taken place at $500{ }^{\circ} \mathrm{C}$ in the coatings which corresponds to the observed reduction in the compressive residual stresses in the coating (Fig. 3). On the other hand, the heat treatment did not lead to significant changes in the grit-blasted region. A possible reason for this clear difference between substrate and coating might be the microstructural differences. Whereas in the substrate clear indication of $\mathrm{Ni}_{3} \mathrm{Nb}$ precipitates can be found, in the coating, the $\mathrm{Nb}$ might only be segregated in solid solution in interdendritic regions without formation of a hardening phase. This is expected to lead to lower strength, and hence easier relaxation in the coating area (Ref 30$)$. The increased mechanical properties of the substrate especially after the grit blasting process might also lead to a lower deformability which might have a negative effect on the bonding of the cold spray coating.

For the interpretation of the strength data, also the quality of the interface is important. Compared to Fig. 10, a lower resolution analysis by SEM is used to analyze a larger area of the interface. Figure 11 shows the comparison of the interface of a coating in the as-sprayed
Fig. 10 SEM micrographs of the substrate-coating interface showing the effect of the 1-h annealing at $500{ }^{\circ} \mathrm{C}$ : (a) assprayed; (b) after annealing
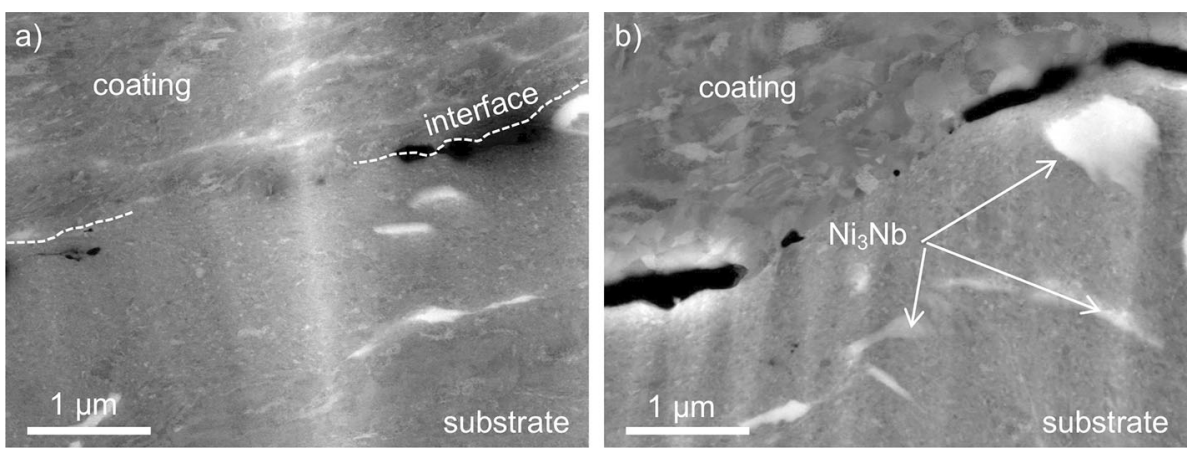


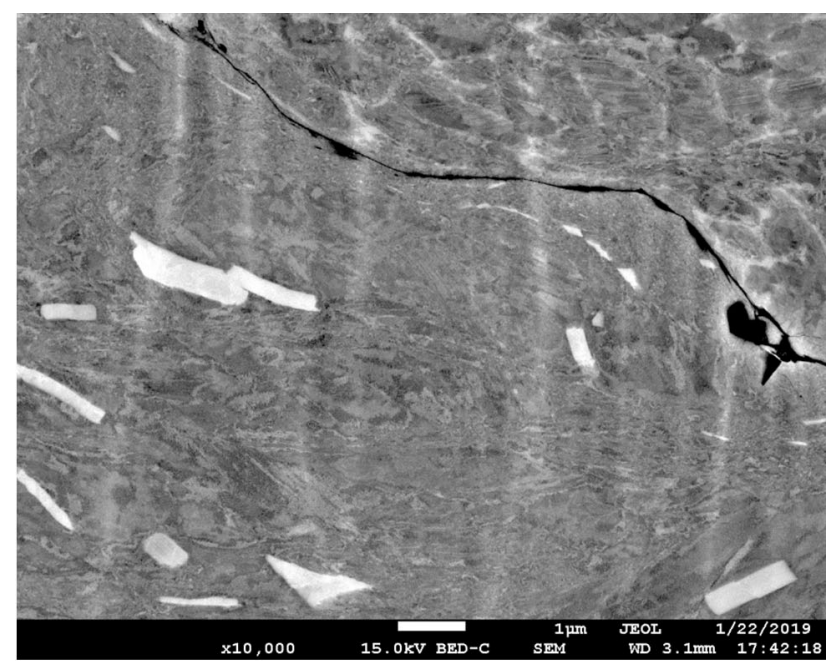

(a)

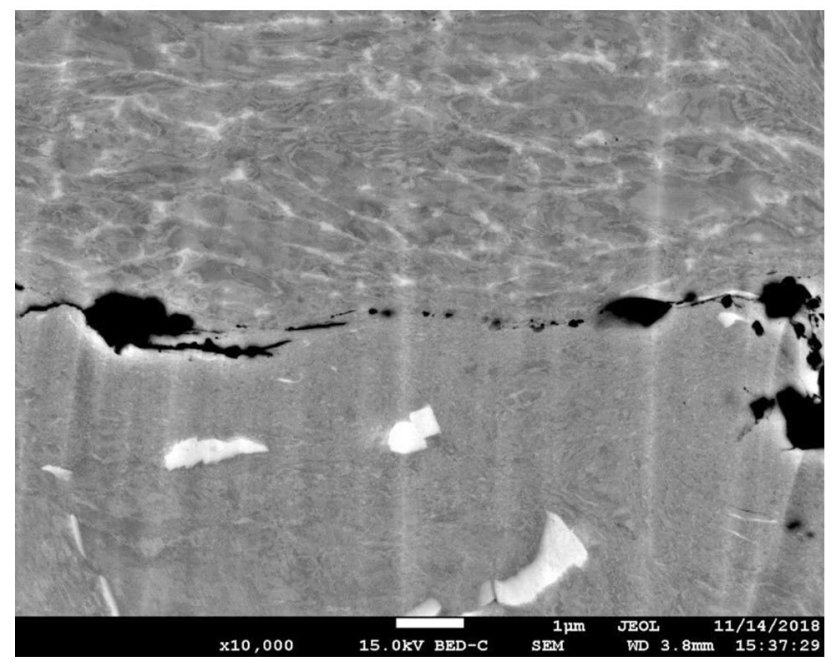

(b)

Fig. 11 SEM micrographs of the interface region of coating (top) and substrate (bottom), left as-sprayed, right annealed conditions $\left(500{ }^{\circ} \mathrm{C}\right.$, $1 \mathrm{~h}$ ). The dark lines are cracks, the larger black areas are remainings from the grit blasting process $\left(\mathrm{Al}_{2} \mathrm{O}_{3}\right)$, and smaller spherical features are pores

condition and after annealing. In the as-sprayed condition, cracks are found which indicate a not optimal bonding between coating and substrate. As only a few cracks are found in the coating itself, this might be related to the substrate properties. As discussed before, the surface region of the substrate shows highly deformed regions which might influence the bonding due to adiabatic shear instabilities as the deformability of both particle and substrate is needed to obtain effective intermixing and formation of sufficient bonding. In addition, also alumina inclusions from the grit blasting process are present, which are expected to further reduce the adhesion strength.
In addition to the microstructural investigations, also an estimation of the interfacial toughness by a method proposed by Chicot et al (Ref 31) has been made. As the samples were embedded and additionally the coatings were rather thin, no quantitative values can be determined. However, a comparison of the different coatings showed a rather high scattering of the interfacial toughness. This was mainly attributed to the large and varying amounts of alumina grits from the grit blasting process at the interface, which promotes crack growth and reduce by that interfacial toughness.

Depositing a thicker layer on the substrate also implies that the propelling gases are longer in contact with the substrate. In order to evaluate if that might have an effect on the microstructure or the stress state simulations by using the KSS software (Ref 32) have been made. The results indicate that the propellant gas is only slightly above $300{ }^{\circ} \mathrm{C}$ at the substrate location. This is not expected to heat the substrate much above $300{ }^{\circ} \mathrm{C}$ and have a significant effect on the coating characteristics. This also corresponds with temperature measurements during the deposition which confirm temperatures below $350{ }^{\circ} \mathrm{C}$. In addition, the simulation gave a compressive residual stress state of $-192 \mathrm{MPa}$ which is in rather good accordance with the results presented here.

In conclusion, the findings support the assumption that the energy release rate in the coatings is controlling the adhesion strength. Therefore, annealed samples with low residual stress levels showed high-strength values. Scattering of the data is at least partially a result of the grit blasting process, which turns out to have negative effects on the bonding. In addition, it is worthwhile to mention that the microstructure in the coatings is significantly different compared to casted substrates, which are related to the microstructure of the gas atomized powder.

\section{Conclusions}

INCONEL 718 was deposited on INCONEL 718 substrates by cold gas spraying. The coating showed a reduced adhesion strength with increasing thickness. As microstructural features and also the residual stress depth profiles were similar in the three investigated coatings, it can be concluded that the increased energy release rate in the thicker coatings leads to the reduced adhesion strength. The scatter of the data was related to the grit blasting process, as alumina grits are found at the interface in a relatively unpredictable manner. In addition, the bonding to the substrate might be negatively influenced by the high deformation induced in the substrate due to the grit blasting. Furthermore, the microstructure of the coating is 
significantly different from the substrate as $\mathrm{Ni}_{3} \mathrm{Nb}$ precipitates are missing.

After a heat treatment at $500{ }^{\circ} \mathrm{C}$ for $1 \mathrm{~h}$, the residual stress in the coatings relaxed, whereas the residual stress in the substrate remained. The reduced coating residual stress led to a significant improvement of the adhesion stress.

Acknowledgements Open Access funding provided by Projekt DEAL. The authors would like to express their thanks to Mr. KarlHeinz Rauwald for his help to operate the cold gas facility. The authors also acknowledge using ZGH infrastructure (Hitachi IM400Plus cross-section polisher and JEOL JSM-7200F SEM/EDX/ EBSD) at the Ruhr University Bochum.

Open Access This article is licensed under a Creative Commons Attribution 4.0 International License, which permits use, sharing, adaptation, distribution and reproduction in any medium or format, as long as you give appropriate credit to the original author(s) and the source, provide a link to the Creative Commons licence, and indicate if changes were made. The images or other third party material in this article are included in the article's Creative Commons licence, unless indicated otherwise in a credit line to the material. If material is not included in the article's Creative Commons licence and your intended use is not permitted by statutory regulation or exceeds the permitted use, you will need to obtain permission directly from the copyright holder. To view a copy of this licence, visit http://creativecommons. org/licenses/by/4.0/.

\section{References}

1. H. Assadi, H. Kreye, F. Gärtner, and T. Klassen, Cold SprayingA Materials Perspective, Acta Mater., 2016, 116, p 382-407

2. V. Champagne and D. Helfritch, The Unique Abilities of Cold Spray Deposition, Int. Mater. Rev., 2016, 61, p 437-455

3. C.M. Kay and J. Karthikeyan, High Presure Cold Spray, Principles and Applications, ASM International, Materials Park, $\mathrm{OH}$, 2016

4. R.N. Raoelison, Y. Xie, T. Sapanathan, M.P. Planche, R. Kromer, S. Costil, and C. Langlade, Cold Gas Dynamic Spray Technology: A Comprehensive Review of Processing Conditions for Various Technological Developments Till to Date, Addit. Manuf., 2017, 19, p 134-159

5. T. Schmidt, H. Assadi, F. Gärtner, H. Richter, T. Stoltenhoff, H. Kreye, and T. Klassen, From Particle Acceleration to Impact and Bonding in Cold Spraying, J. Therm. Spray Technol., 2009, 18, p 794

6. M. Grujicic, C.L. Zhao, W.S. DeRosset, and D. Helfritch, Adiabatic Shear Instability Based Mechanism for Particles/Substrate Bonding in the Cold-Gas Dynamic-Spray Process, Mater. Des., 2004, 25, p 681-688

7. W.Y. Li, C. Zhang, X. Guo, J. Xu, C.J. Li, H. Liao, C. Coddet, and K.A. Khor, Ti and Ti-6Al-4V Coatings by Cold Spraying and Microstructure Modification by Heat Treatment, Adv. Eng. Mater., 2007, 9, p 418-423

8. V. Luzin, K. Spencer, and M.X. Zhang, Residual Stress and Thermo-mechanical Properties of Cold Spray Metal Coatings, Acta Mater., 2011, 59, p 1259-1270

9. V. Champagne, M. Trexler, D. Helfritch, P. Leyman, and B. Gabriel, Repair of aircraft materials by cold spray. in Aeromat (2010)
10. C.A. Widener, O.C. Ozdemir, and M. Carter, Structural Repair Using Cold Spray Technology for Enhanced Sustainability of High Value Assets, Procedia Manuf, 2018, 21, p 361-368

11. T.M. Pollock and S. Tin, Nickel-Based Superalloys for Advanced Turbine Engines: Chemistry, Microstructure and Properties, J. Propuls. Power, 2006, 22, p 361-374

12. W. Wong, E. Irissou, P. Vo, M. Sone, F. Bernier, J.G. Legoux, H. Fukanuma, and S. Yue, Cold Spray Forming of Inconel 718, J. Therm. Spray Technol., 2012, 22, p 413-421

13. W.T. Sun, A.W.Y. Tan, A. Bhowmik et al., Deposition Characteristics of Cold Sprayed Inconel 718 Particles on Inconel 718 Substrates with Different Surface Conditions, Mater. Sci. Eng. A Struct. Mater. Prop. Microstruct. Process., 2018, 720, p 75-84

14. D. Levasseur, S. Yue, and M. Brochu, Pressureless Sintering of Cold Sprayed Inconel 718 Deposit, Mater. Sci. Eng. A, 2012, 556, p 343-350

15. X.T. Luo, M.L. Yao, N. Ma, M. Takahashi, and C.J. Li, Deposition Behavior, Microstructure and Mechanical Properties of an In-Situ Micro-Forging Assisted Cold Spray Enable Additively Manufactured Inconel 718 Alloy, Mater. Des., 2018, 155, p 384395

16. W. Ma, Y. Xie, C. Chen, H. Fukanuma, J. Wang, Z. Ren, and R. Huang, Microstructural and Mechanical Properties of High-Performance Inconel 718 Alloy by Cold Spraying, J. Alloys Compd., 2019, 792, p 456-467

17. L.I. Pérez-Andrade, F. Gärtner, M. Villa-Vidaller, T. Klassen, J. Muñoz-Saldaña, and J.M. Alvarado-Orozco, Optimization of Inconel 718 Thick Deposits by Cold Spray Processing and Annealing, Surf. Coat. Technol., 2019, 378, 124997

18. R. Singh, S. Schruefer, S. Wilson, J. Gibmeier, and R. Vassen, Influence of Coating Thickness on Residual Stress and AdhesionStrength of Cold-Sprayed Inconel 718 Coatings, Surf. Coat. Technol., 2018, 350, p 64-73

19. R. Singh, K.-H. Rauwald, E. Wessel, G. Mauer, S. Schruefer, A. Barth, S. Wilson, and R. Vassen, Effects of Substrate Roughness and Spray-Angle on Deposition Behavior of Cold-Sprayed Inconel 718, Surf. Coat. Technol., 2017, 319, p 249-259

20. A.B.S. Wilson, M. Nestler, and S. Kudapa, Cold gas spray coating methods and compositions, patent application, $\mathrm{W} \mathrm{O}$ 2017/003427 A 1 (2015)

21. G. Mauer, R. Singh, K.-H. Rauwald, S. Schrüfer, S. Wilson, and R. Vaßen, Diagnostics of Cold-Sprayed Particle Velocities Approaching Critical Deposition Conditions, J. Therm. Spray Technol., 2017, 26, p 1423-1433

22. ISO, 6892-1, Metallic Materials-Tensile Testing-Part 1, Method of Test at Room Temperature, in, Beuth Verlag (2016).

23. E. Obelode and J. Gibmeier, Influence of the Interfacial Roughness on Residual Stress Analysis of Thick Film Systems by Incremental Hole Drilling, Mater. Sci. Forum, 2014, 768-769, p 136-143

24. N.J. Rendler and I. Vigness, Hole-Drilling Strain-Gage Method of Measuring Residual Stresses, Exp. Mech., 1996, 6, p 577-586

25. E. Held and J. Gibmeier, Residual Stress Analysis of Thick Film Systems by the Incremental Hole-Drilling Method-Influence of Interlayers and Interfacial Roughness, HTM J. Heat Treat. Mater., 2014, 69, p 71-79

26. B. Eigenmann and E. Macherauch, Röntgenographische Untersuchung von Spannungszuständen in Werkstoffen Teil IV, Materialwiss Werkstoff, 1996, 27, p 491-501

27. Y.C. Tsui and T.W. Clyne, An Analytical Model for Predicting Residual Stresses in Progressively Deposited Coatings Part 1: Planar Geometry, Thin Solid Films, 1997, 306, p 23-33

28. M. Multigner, S. Ferreira-Barragáns, E. Frutos, M. Jaafar, J. Ibáñez, P. Marín, M.T. Pérez-Prado, G. González-Doncel, A. Asenjo, and J.L. González-Carrasco, Superficial Severe Plastic Deformation of 316 LVM Stainless Steel Through Grit Blasting: 
Effects on its Microstructure and Subsurface Mechanical Properties, Surf. Coat. Technol., 2010, 205, p 1830-1837

29. Y. Zou, W. Qin, E. Irissou, J.-G. Legoux, S. Yue, and J.A. Szpunar, Dynamic Recrystallization in the Particle/Particle Interfacial Region of Cold-Sprayed Nickel Coating: Electron Backscatter Diffraction Characterization, Scripta Mater., 2009, 61, p 899-902

30. M.A.C. Slama, Structural Characterization of the Aged Inconel 718, J. Alloys Compd., 2000, 306, p 277-284
31. D.D. Chicot, P. Démarécaux, and J. Lesage, Apparent Interface Toughness of Substrate and Coating Couples from Indentation Tests, Thin Solid Films, 1996, 283, p 151-157

32. https://kinetic-spray-solutions.com/kssapp/.

Publisher's Note Springer Nature remains neutral with regard to jurisdictional claims in published maps and institutional affiliations. 